\title{
Exposed bright features on the comet 67P/Churyumov-Gerasimenko: distribution and evolution
}

\author{
J. D. P. Deshapriya ${ }^{1}$, M. A. Barucci ${ }^{1}$, S. Fornasier ${ }^{1}$, P. H. Hasselmann ${ }^{1}$, C. Feller ${ }^{1}$, H. Sierks ${ }^{2}$, A. Lucchetti ${ }^{3}$, \\ M. Pajola ${ }^{4}$, N. Oklay ${ }^{5}$, S. Mottola ${ }^{5}$, N. Masoumzadeh ${ }^{2}$, C. Tubiana ${ }^{2}$, C. Güttler ${ }^{2}$, C. Barbieri' ${ }^{6}$, P. L. Lamy ${ }^{7}$, \\ R. Rodrigo ${ }^{8,9}$, D. Koschny ${ }^{10}$, H. Rickman ${ }^{11,12}$, J.-L. Bertaux ${ }^{13}$, I. Bertini ${ }^{6}$, D. Bodewits ${ }^{14}$, S. Boudreault ${ }^{2}$, \\ G. Cremonese ${ }^{3}$, V. Da Deppo ${ }^{15}$, B. J. R. Davidsson ${ }^{16}$, S. Debei ${ }^{17}$, M. De Cecco ${ }^{18}$, J. Deller ${ }^{2}$, M. Fulle ${ }^{19}$, \\ O. Groussin ${ }^{8}$, P. J. Gutierrez ${ }^{20}$, H. V. Hoang ${ }^{1}$, S. F. Hviid ${ }^{5}$, W. Ip ${ }^{21}$, L. Jorda ${ }^{8}$, H. U. Keller ${ }^{22}$, J. Knollenberg ${ }^{5}$, \\ R. Kramm², E. Kührt ${ }^{5}$, M. Küppers ${ }^{23}$, L. Lara ${ }^{20}$, M. Lazzarin ${ }^{6}$, J. J. Lopez Moreno ${ }^{20}$, F. Marzari ${ }^{6}$, \\ G. Naletto ${ }^{15,24,25}$, F. Preusker ${ }^{5}$, X. Shi ${ }^{2}$, N. Thomas ${ }^{26}$, and J.-B. Vincent ${ }^{5}$
}

(Affiliations can be found after the references)

Received 17 October 2017 / Accepted 28 December 2017

\begin{abstract}
Context. Since its arrival at the comet 67P/Churyumov-Gerasimenko in August 2014, the Rosetta spacecraft followed the comet as it went past the perihelion and beyond until September 2016. During this time there were many scientific instruments operating on board Rosetta to study the comet and its evolution in unprecedented detail. In this context, our study focusses on the distribution and evolution of exposed bright features that have been observed by OSIRIS, which is the scientific imaging instrument aboard Rosetta. Aims. We envisage investigating various morphologies of exposed bright features and the mechanisms that triggered their appearance. Methods. We co-registered multi-filter observations of OSIRIS images that are available in reflectance. The Lommel-Seeliger disk function was used to correct for the illumination conditions and the resulting colour cubes were used to perform spectrophotometric analyses on regions of interest.

Results. We present a catalogue of 57 exposed bright features observed on the nucleus of the comet, all of which are attributed to the presence of $\mathrm{H}_{2} \mathrm{O}$ ice on the comet. Furthermore, we categorise these patches under four different morphologies and present geometric albedos for each category.

Conclusions. Although the nucleus of 67P/Churyumov-Gerasimenko appears to be dark in general, there are localised $\mathrm{H}_{2} \mathrm{O}$ ice sources on the comet. Cometary activity escalates towards the perihelion passage and reveals such volatile ices. We propose that isolated $\mathrm{H}_{2} \mathrm{O}$ ice patches found in smooth terrains in regions, such as Imhotep, Bes, and Hapi, result from frost as an aftermath of the cessation of the diurnal water cycle on the comet as it recedes from perihelion. Upon the comet's return to perihelion, such patches are revealed when sublimation-driven erosion removes the thin dust layers that got deposited earlier. More powerful activity sources such as cometary outbursts are capable of revealing much fresher, less contaminated $\mathrm{H}_{2} \mathrm{O}$ ice that is preserved with consolidated cometary material, as observed on exposed patches resting on boulders. This is corroborated by our albedo calculations that attribute higher albedos for bright features with formations related to outbursts.
\end{abstract}

Key words. comets: individual: 67P/Churyumov-Gerasimenko - techniques: photometric - methods: data analysis

\section{Introduction}

Upon its rendezvous with the Jupiter-family comet 67P/Churyumov-Gerasimenko (hereafter 67P) in August 2014, the Rosetta spacecraft carried out various studies about the cometary nucleus and coma using its onboard scientific instruments until the end of the extended mission in September 2016. Rosetta encountered 67P during the inbound phase of its orbit and followed its evolution past perihelion, as it was on its way to aphelion. The perihelion passage of the comet took place on 13 August 2015 at a heliocentric distance of 1.24 AU. During this phase the spacecraft was required to remain at a safe distance of hundreds of $\mathrm{km}$ from the nucleus because of the increased cometary activity. However, Rosetta managed to get closer to the surface many times during its mission and to perform some fly-bys as well. Throughout the mission, the Optical, Spectroscopic and Infrared Remote Imaging System (OSIRIS; Keller et al. 2007) proved to be a fundamental instrument aboard Rosetta, providing a detailed characterisation of the cometary nucleus in exceptional spatial resolution. The OSIRIS instrument was composed of two cameras, called Narrow Angle Camera (NAC) and Wide Angle Camera (WAC). The NAC was equipped with 11 broad band filters in the wavelength range of 269-989 $\mathrm{nm}$ and was generally used for the observation of the nucleus, while the WAC had 14 narrow band filters in the wavelength range from $246-631 \mathrm{~nm}$ and was intended for the observation of the dust grains and gaseous species in the coma.

While all previous cometary space missions have been brief fly-bys that only enabled a transient acquisition of data, Rosetta was able to further scientific research in an unprecedented manner by observing the comet at high spatial resolution continuously for more than $2 \mathrm{yr}$. The OSIRIS cameras permitted the observation of an irregular nucleus made up of two cometesimals forming a bilobate shape that hosts a plethora of heterogeneous geomorphological features (Sierks et al. 2015; Thomas et al. 2015). 67P has a low geometric albedo of $6.5 \pm 0.2 \%$ at $649 \mathrm{~nm}$ 

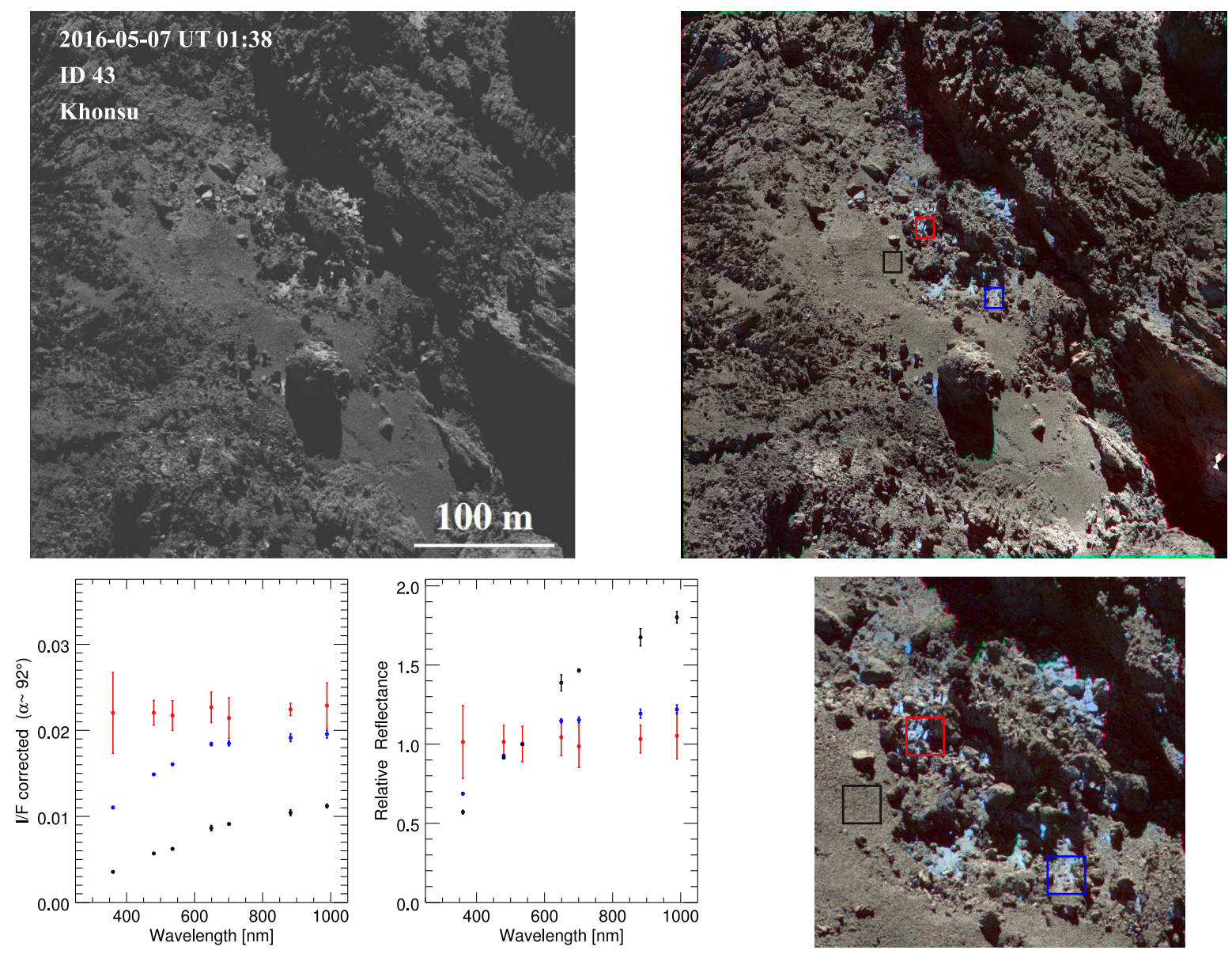

Fig. 1. Top left: narrow angle camera monochromatic image ( $F 22$ filter centred at $649.2 \mathrm{~nm}$ ) revealing a part of the Khonsu region on the comet where bluish material and exposed bright patches are present in the form of a cluster. Right: an $R G B$ image produced with the STIFF (Bertin 2012) program using the NAC filters centred at wavelengths $882.1,649.2$, and $480.7 \mathrm{~nm}$ for red, green and blue colours. This image also depicts 3 regions of interest chosen for the spectrophotometric analysis. Bottom left: spectrophotometric plots of the aforementioned regions of interest are shown. Right: a zoom onto the 3 regions of interest on the $R G B$ image is represented. The red box refers to an exposed bright patch (Deshapriya et al. 2016) containing $\mathrm{H}_{2} \mathrm{O}$ ice, whose reflectance has been sampled $(1 \times 2$ pixels per filter) with scrutiny to avoid saturated pixels and the blue box corresponds to $\mathrm{H}_{2} \mathrm{O}$ ice mixed with dust and material from the nucleus $(2 \times 2$ pixels $)$, whereas the black box $(2 \times 2$ pixels $)$ represents the typical nucleus. The dimensions of the boxes are deliberately exaggerated in order to point out their respective locations on the terrain. The red and green features that sometimes appear adjacent to the shadows and near the frame edges in the $R G B$ images are artefacts arising from the imperfect co-registration/receding shadows of the images.

and its surface shows a large colour heterogeneity (Fornasier et al. 2015). Both seasonal and diurnal changes were identified in terms of spectrophotometry and spectral slope analyses (Fornasier et al. 2016).

Although comets are supposed to be made of ice and dust, OSIRIS data have revealed that ice is not observed ubiquitously on the comet. Results by Fornasier et al. (2016) indicated that the cometary surface is covered by an optically thick layer of dust that prevents the direct observation of subsurface ice on the comet, which corroborates previous theoretical (Fanale \& Salvail 1984) and experimental (Sears et al. 1999) models about cometary nuclei. When 67P approached perihelion, there was a substantial rise of cometary activity that progressively removed this layer of dust, allowing the observation of an ice-rich subsurface layer. These authors proposed that the dust layer got thicker following the perihelion passage as the cometary activity gradually ceased. Such is the evidence that the cometary surface is rich in volatile ices often mixed with dust, whose presence can be validated with spectrophotometric, spectral slope analyses, and false-colour $R G B$ images (see Oklay et al. 2017). As shown in spectrophotometric plots of Fig. 1, such volatile-rich terrains (blue dots) are characterised by a red spectral (gradual increase of reflectance with the wavelength) behaviour in their absolute reflectance $(I / F)$. They are characterised also by a higher absolute reflectance than that of the typical nucleus (black dots) for all the wavelengths. Consequently, their albedo is higher than that of the typical nucleus. As far as the relative reflectance is concerned, these terrains have spectrophotometric characteristics similar to the typical nucleus from near ultraviolet wavelengths up to around $650 \mathrm{~nm}$, and then they deviate from the typical nucleus towards infrared wavelengths, diverging with a lower gradient. Another distinction of these volatile-rich terrains is that they appear in a bluish colour in $R G B$ images, as evidenced in Fig. 1, which also reveals the presence of exposed bright patches that can be distinguished from volatile ices mixed with dust by virtue of their characteristic flat spectrophotometric behaviour (represented by red dots therein). Although the $R G B$ image is not ideal for distinguishing between the two, their spectrophotometry clearly identifies the exposed volatile ices. Nevertheless, a careful inspection of the $R G B$ images shows that the exposed ices are identifiable with a dark bluish or whitish colour in $R G B$ images. 
The OSIRIS instrument has revealed exposed bright features that have been attributed to the presence of $\mathrm{H}_{2} \mathrm{O}$ ice in several studies accompanied by multi-filter observations to perform spectrophotometric studies. Analysing the data in the inbound orbit of the comet from 3.6 to 3.0 AU, shortly after the arrival of Rosetta at 67P, Pommerol et al. (2015) reported various features of metre-sized exposed $\mathrm{H}_{2} \mathrm{O}$ ice, belonging to three different categories. Barucci et al. (2016) performed a collective study of eight exposed bright features initially identified with OSIRIS spectrophotometry, coupled with complementary infrared data from Visible and InfraRed Thermal Imaging Spectrometer (VIRTIS-M) spectro-imager, to confirm the presence of $\mathrm{H}_{2} \mathrm{O}$ ice using the $\mathrm{H}_{2} \mathrm{O}$ ice absorption band at $2 \mu \mathrm{m}$. Filacchione et al. (2016a) studied two bright features using VIRTIS-M infrared spectra, attributing their presence to $\mathrm{H}_{2} \mathrm{O}$ ice, making use of the $\mathrm{H}_{2} \mathrm{O}$ ice absorption bands at $1.05,1.25,1.5$, and $2.0 \mu \mathrm{m}$. Filacchione et al. (2016b) put forward the presence of exposed $\mathrm{CO}_{2}$ ice identified for the first time on a cometary surface using the absorption bands at 1.97, 2.01, 2.07, 2.7, and $2.78 \mu \mathrm{m}$ of VIRTIS-M infrared spectra. Following about a month from this detection, Fornasier et al. (2016) reported the presence of two large bright patches (about $1500 \mathrm{~m}^{2}$ each) almost at the original $\mathrm{CO}_{2}$ location, which indicates the local heterogeneity of the distribution of volatile ices in the comet. Oklay et al. (2016) discussed several previously studied exposed bright features and studied several new features as well. Deshapriya et al. (2016) focussed their study on four exposed $\mathrm{H}_{2} \mathrm{O}$ ice features on the Khonsu region on the southern hemisphere of the comet. Pajola et al. (2017) observed the freshly exposed interior of the comet $67 \mathrm{P}$, following a wide collapse and estimated an albedo of $40 \%$ (lower limit) for the exposed material.

In this context, we report a comprehensive study of exposed bright features observed by the OSIRIS instrument and characterised by flat spectrophotometric curves as seen in Fig. 1, during the whole time spent at 67P. In Appendix A, we catalogue such features from early August 2014 (3.6 AU inbound) through the perihelion passage in August 2015 (1.24 AU) up to the end of September 2016 (3.8 AU outbound), taking into account their morphology and temporal evolution as the heliocentric distance of the comet was changing. In addition, we made use of the images recorded by Rosetta Navigation Camera (NAVCAM) to refine the temporal evolution of some bright features. The objective of this study is to better constrain the morphologies hosting these bright features, to investigate potential triggers for their appearance and to monitor their chronological evolution when multiple observations are available. Therefore, this paper is structured as follows. In Sect. 2, the data set and methods are presented; Sect. 3 details the catalogue of exposed bright features under investigation. Then, in Sect. 4, we present our study on the exposed bright features, which is followed by their spectrophotometric analysis and albedo calculations in Sect. 5. A general discussion is presented in the Sect. 6 and, finally, Sect. 7 is dedicated to the conclusions drawn from this work.

\section{Data and methods}

We made use of the data available in level $3 \mathrm{~B}$ of the OSIRIS standard data reduction pipeline throughout this work. These data are based on level B data, which underwent several corrections for bias, flat-field, geometric distortion, and calibration in absolute flux as detailed in Küppers et al. (2007) and Tubiana et al. (2015), and are expressed in the form of spectral radiance with $\mathrm{W} \mathrm{sr}^{-1} \mathrm{~m}^{-2} \mathrm{~nm}^{-1}$ as units. Then the data are converted to the radiance factor $(I / F)$ in level 3B according to Eq. (1), which is written as

Radiance factor $(I / F)=\frac{I(i, e, \alpha, \lambda)}{F(\lambda)}$,

where $I$ is referred to the observed scattered spectral radiance, and $F$ is the solar spectral irradiance at the heliocentric distance of the comet, divided by $\pi$. The symbols $i, e, \alpha$, and $\lambda$ correspond to the incidence angle, emission angle, phase angle, and wavelength, respectively. The solar irradiance has been calculated at the central wavelength of each filter to be consistent with the derivation of the absolute calibration factors.

Because the image acquisitions are not made simultaneously, but with a lag of few seconds to switch the filter wheel, the images of a given sequence do not show exactly the same field of view; a sequence of observations can be made with a minimum of 2 filters up to 11 filters in the case of NAC. This is due to both the motion of the spacecraft and the cometary rotation during the image acquisition. In order to take this effect into account and perform accurate spectrophotometry, we adopted the image co-registration algorithm as in Deshapriya et al. (2016). We consequently obtained a data cube with dimensions corresponding to the number of filters available in the observation sequence.

We then computed the ratio between the radiance factor and the Lommel-Seeliger disk function (Fairbairn 2005) to account for the various illumination conditions prevailing during the observations. The Lommel-Seeliger disk function $D$ is defined in Eq. (2). The incident and emission angles required for the calculation were calculated using a shape model of the comet.

$$
D(i, e)=\frac{2 \cos (i)}{\cos (i)+\cos (e)} .
$$

\section{Catalogue of exposed bright features}

During the early observations of the nucleus of 67P, Pommerol et al. (2015) introduced three types of exposed bright features whereas our study expands the types of distinguishable bright features to five as follows:
1. Isolated patches on smooth terrains
2. Isolated patches close to irregular structures
3. Patches resting on boulders
4. Clusters of patches
5. Frost

Each of these types is described in detail in Sect. 4. We highlight that we have mentioned frost because it shows the characteristic spectrophotometric properties of the $\mathrm{H}_{2} \mathrm{O}$ ice in OSIRIS multi-filter observations. Nevertheless, we did not include frost in our catalogue of bright features owing to its ephemeral nature (lifetime of few minutes around the perihelion passage; see Fornasier et al. 2016) and inherent distinction in the process of formation compared with exposed $\mathrm{H}_{2} \mathrm{O}$ ice observed on 67P. Therefore, we make it clear that our catalogue of bright features does not contain frost, but only first four types of the above list.

In the preparation of this catalogue, we established a definition of an exposed bright feature attributed to $\mathrm{H}_{2} \mathrm{O}$ ice by adhering to the following criteria :

1. A ratio of at least 1.5 between the reflectances of bright features and the typical nucleus for a given filter.

2. The presence of a flat spectrophotometric curve in reflectance. 


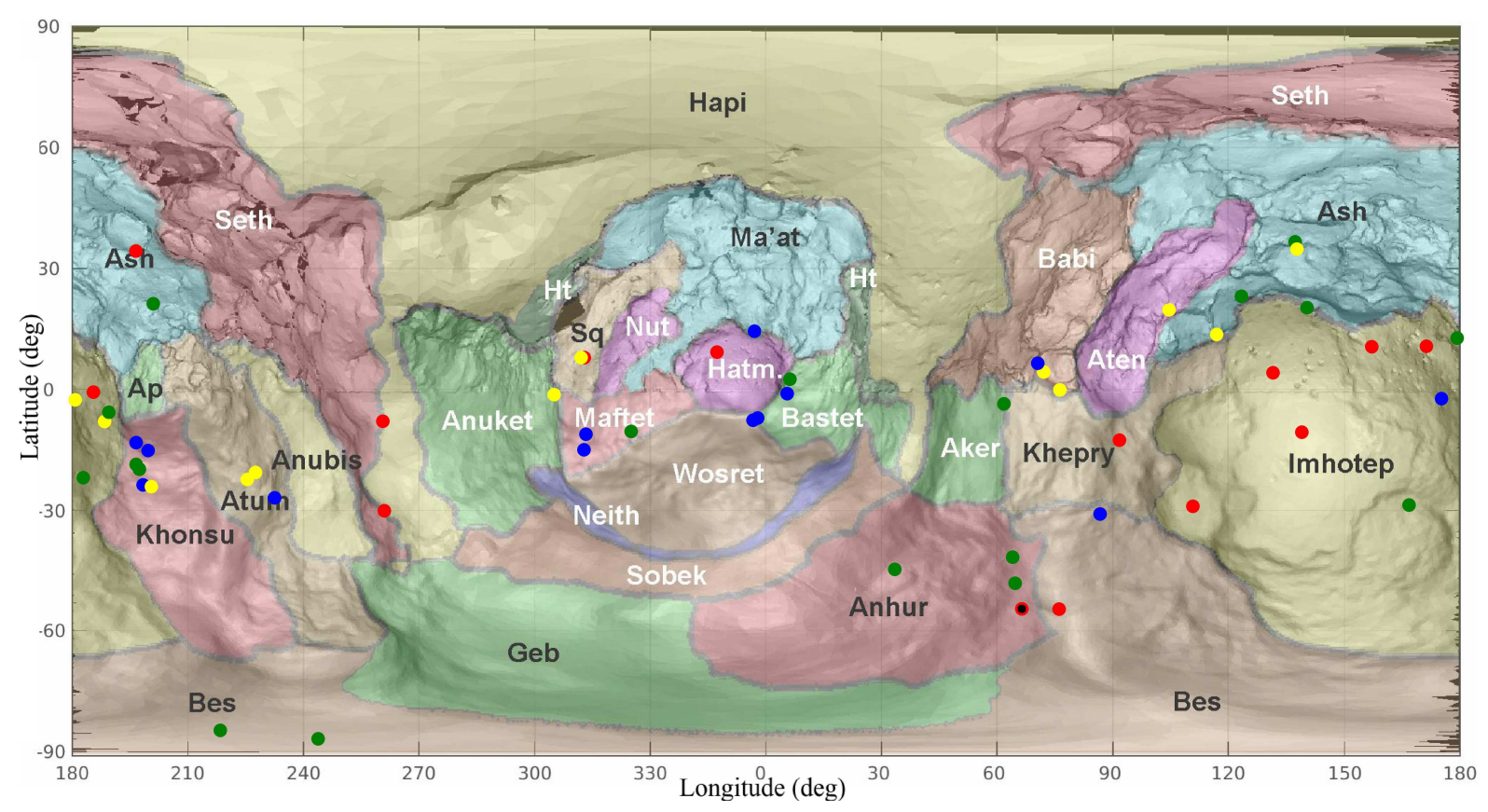

Fig. 2. Map of bright features observed on 67P. The detections of $\mathrm{H}_{2} \mathrm{O}$ ice are indicated by red, green, blue, and yellow colours, which correspond to the feature types 1-4, respectively; the black dot indicates the unique detection of $\mathrm{CO}_{2}$ ice (Filacchione et al. 2016b) where $\mathrm{H}_{2} \mathrm{O}$ ice was detected few weeks later (Fornasier et al. 2016). This map is the cylindrical projection of a merged shape model resulting from the shape models SHAP4S (Preusker et al. 2015) and SHAP5 (Jorda et al. 2016) for the northern hemisphere and southern hemisphere, respectively.

Table 1. Four types of bright features appearing in the catalogue and their respective counts.

\begin{tabular}{lc}
\hline \hline Feature type & Count \\
\hline 1. Isolated patches on smooth terrains & 14 \\
2. Isolated patches close to irregular structures & 18 \\
3. Patches resting on boulders & 13 \\
4. Clusters of patches & 12 \\
\hline Total & 57 \\
\hline
\end{tabular}

As per the first criterion, a ratio of 1.5 would imply that the reflectance of the bright feature is greater than that of the nucleus for all the available filters. If one were to compare different observations made at the same phase angle, a higher ratio would imply that the bright feature in question is fresher and less contaminated. In this study we found cases in which the ratio was sometimes 6 and 13 (for a bright patch in Bes region as shown in Fig. 5f) for different features, implying the presence of fresher and less contaminated $\mathrm{H}_{2} \mathrm{O}$ ice. It is noteworthy that most of the pixels in these cases were saturated and the actual value of the ratio would have been even greater. The second criterion requires that observations be multi-filter sequences, imaged at least with three NAC filters. This allows us to verify whether the candidate feature possesses the flat spectrophotometric curve in reflectance and relative reflectance plots, which is characteristic to the $\mathrm{H}_{2} \mathrm{O}$ ice.

Following the above criteria we found 51 different features of bright patches in the multi-filter observations in which spectrophotometric analyses were possible. In addition to this, we analysed the observations where a single filter was available and present six more features adhering only to the first criterion. In these observations, we used the higher reflectance of the feature compared to the surrounding as the proxy for the $\mathrm{H}_{2} \mathrm{O}$ ice. However we note that the latter is not an exhaustive list. We considered that an individual feature could be composed of an individual patch or a number of patches ( 2 or several smaller patches in the case of clusters). Therefore, the total of 57 features is not an exhaustive measurement of all the individual bright patches we studied. Table 1 lists the four types of bright features catalogued along with their respective counts. Figure 2 shows the locations of bright patches on a cylindrically projected map of the nucleus, favouring equatorial regions for most of the bright features, while the majority of these features are found on the southern latitudes of the comet. However, one should be cautious in interpreting this observation, for this might be an observational bias as it was the southern hemisphere that was mostly observed during the mission. The catalogue of exposed bright features is presented in the Appendix A with corresponding observational circumstances. The numbers $1-51$ refer to the multi-filter observations enabling spectrophotometric analyses in which characteristic flat spectrophotometric curves of $\mathrm{H}_{2} \mathrm{O}$ ice could be used to confirm its presence, whereas the numbers 52-57 refer to the observations with a single filter in which case higher reflectance of $\mathrm{H}_{2} \mathrm{O}$ compared to the surrounding nucleus terrain was used as the proxy. We remark that the observations from 52-57 are not an exhaustive list. The column "Detection" corresponds to the first observation of the feature and the column "Life" gives the number of days that the feature has survived. These days are calculated using the other temporal observations of the same feature and hence they imply a lower limit of real lifetime of the feature. It is also noteworthy that the lifetime of 0 indicates that the feature in question has only been observed once; hence it is not possible to derive a lifetime for such features. Also the area measurements of the clusters of features are not given because the measurements may carry errors due to the presence of a number of smaller individual features present on different morphological structures. 

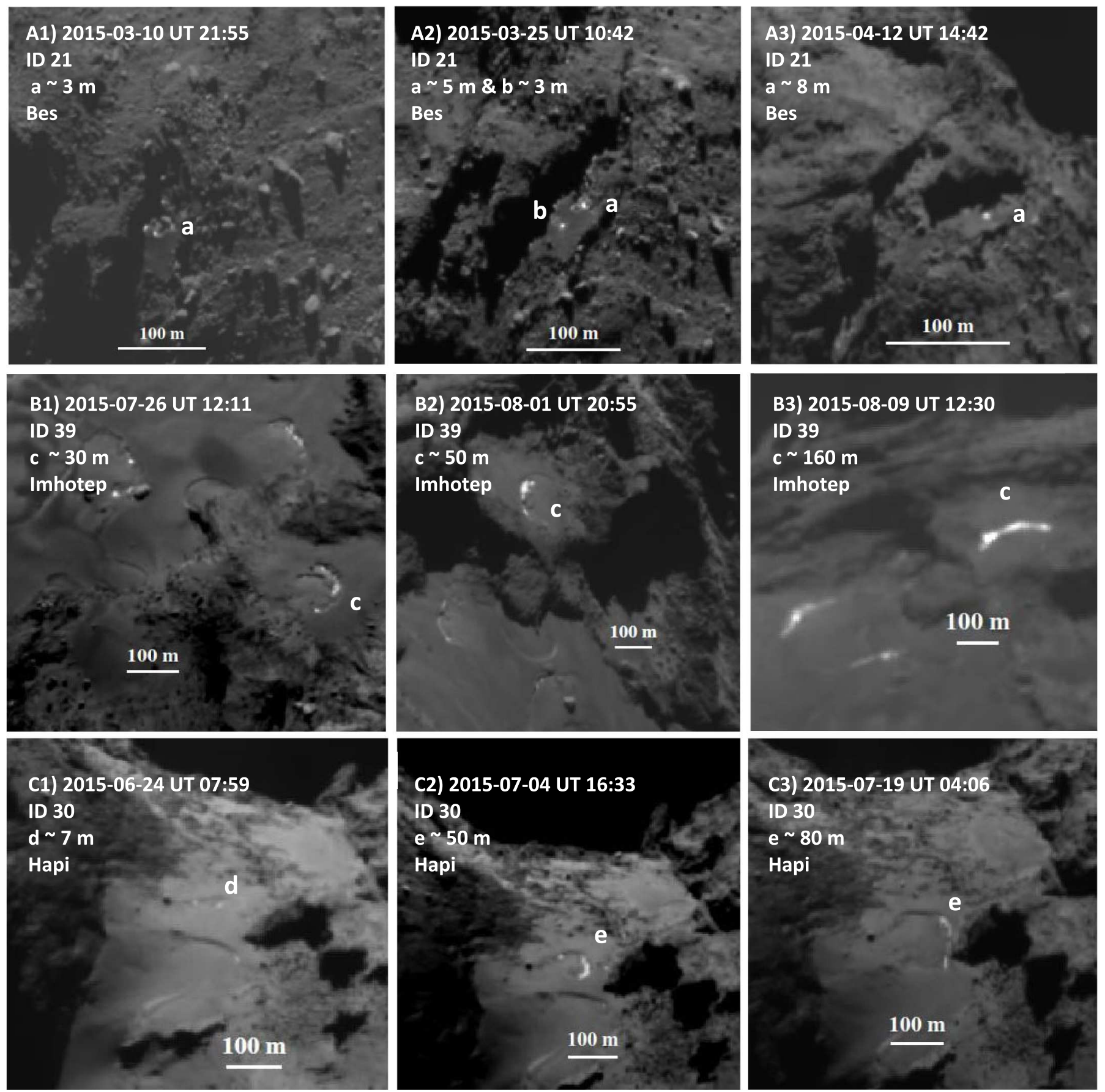

Fig. 3. Temporal variations of 3 isolated bright patches on smooth terrains in Bes, Imhotep, and Hapi regions belonging to the type 1 of exposed bright features. Each panel corresponds to the morphological evolution of a selected patch during the course of the OSIRIS observations.

\section{Types of bright features}

\subsection{Isolated patches on smooth terrains}

This category of bright features encompasses isolated individual bright patches that are located on smooth terrain. These bright patches are likely to be formed by the sublimation-driven erosion (sublimation of volatiles such as $\mathrm{CO}, \mathrm{CO}_{2}$, and $\mathrm{H}_{2} \mathrm{O}$ ices) on the cometary surface that was gradually increasing when $67 \mathrm{P}$ got closer (inbound) to perihelion. Compared to the consolidated cometary material on rugged terrains of the comet, smooth terrains represent dusty material with low tensile strength (Groussin et al. 2015a) that is easily subject to erosion. Hence, cometary erosion was observed mostly on the smooth terrains. In Fig. 3, we illustrate the temporal evolution of isolated bright patches located in Bes, Imhotep, and Hapi regions of the comet. Panel A of Fig. 3 shows an isolated bright patch on the smooth terrain that survived for about a period of 1 month. This patch annotated with "a" is joined by an ephemeral companion patch annotated with "b" in the window A2. The growth of its size is due to increasing erosion.

On the Imhotep region, where a vast majority of smooth terrains are present, large irregular bright patches appeared (Groussin et al. 2015b) just prior to the perihelion passage as erosion was escalating. At the same time on the Hapi region, erosion was also at play (Davidsson et al., in prep.) leading to the observation of isolated bright features. In addition to these 

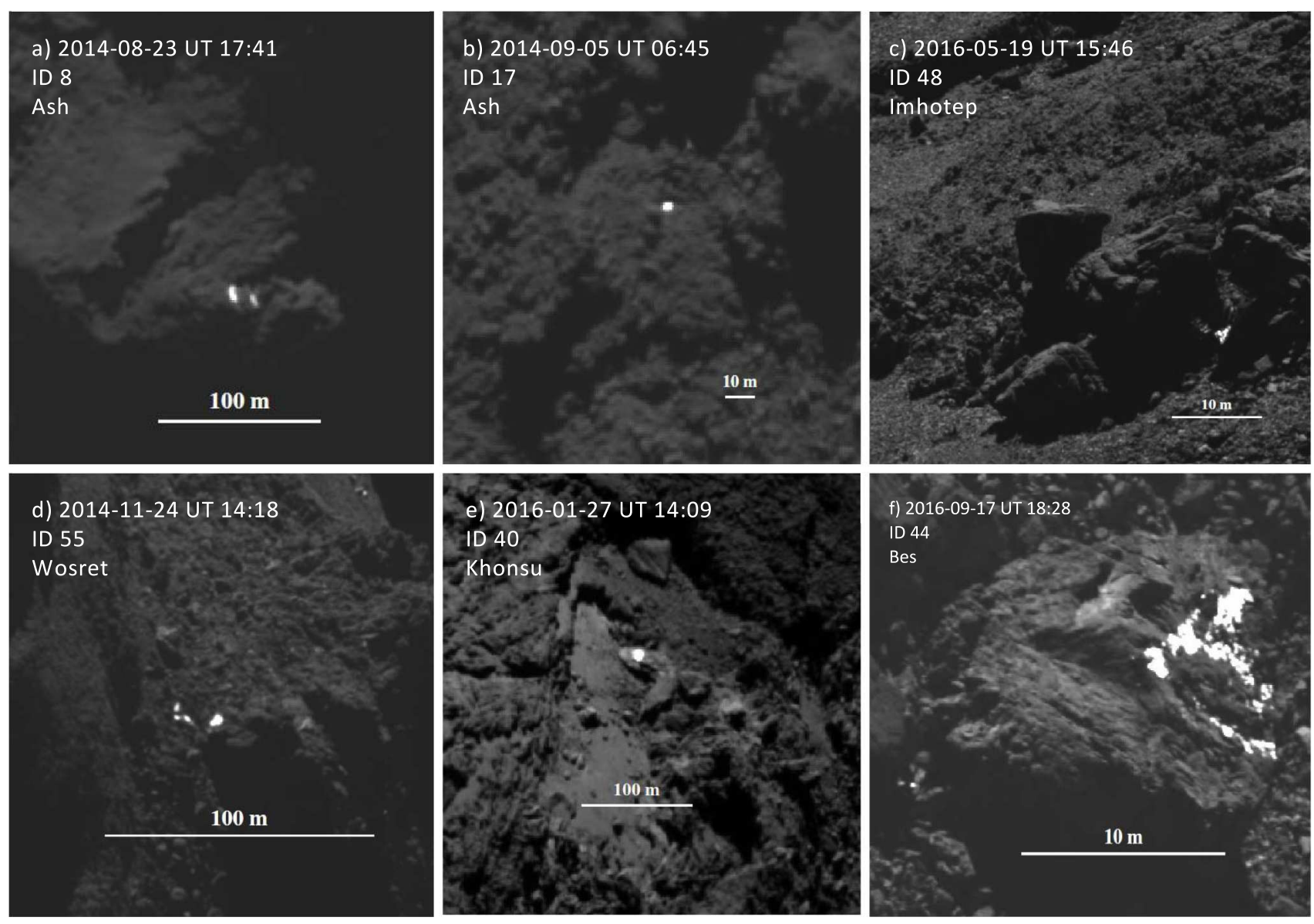

Fig. 4. Top panel: Type 2 of exposed bright features which are the isolated patches close to irregular structures. Bottom panel: Type 3 of exposed bright features which are the patches resting on boulders.

large features, it is noteworthy that several smaller bright features have also been observed on Imhotep towards its boundary, albeit on smooth terrain a few weeks before the perihelion passage. The erosion-driven scarp-retreating processes (El-Maarry et al. 2017) that triggered the appearance of bright patches are clearly observable in the panels B and C of Fig. 3. These patches significantly differ from other patches in this category by their arc-shaped irregular structure and size (length of the arc shape), although they are observed isolated in smooth terrains too. It is very interesting that the patch annotated with "c" in the panel B, almost doubles its dimension within a period of a week, and 1 week later this patch achieves a dimension that is more than five times its size 2 weeks earlier. Besides, this patch "c", the panel B reveals the presence and growth of other subordinate patches in the smooth terrains of Imhotep. Panel " $C$ " brings out the accelerated growth of another patch where a small 7 $\mathrm{m}$ patch emerges and develops into a much larger arc-shaped structure measuring about $80 \mathrm{~m}$ in the course of little more than 3 weeks. Groussin et al. (2015b) showed that this observed erosion in Imhotep could not be solely due to sublimation of volatile species such as $\mathrm{H}_{2} \mathrm{O}$ and $\mathrm{CO}_{2}$ at their corresponding heliocentric distances and that it could be the low tensile strength of the cometary material (Groussin et al. 2015a) or the processes such as clathrate destabilisation or amorphous water ice crystallisation, which would result in the observed rates of erosion.

In our catalogue of bright patches, we have 14 different features under this category. One interesting remark in this category is that except for one feature, all the other isolated patches on the smooth terrains have been observed from early March to early August 2015. This implies that all of these patches have appeared before the perihelion passage. However, there is an observational bias coupled with this remark, as Rosetta was obliged to have distant orbits around the comet in order to prevent potential damage during the period of perihelion passage due to rising cometary activity. Rosetta resumed its closer orbits around the comet in January 2016 and we have not been able to trace any more isolated patches on the smooth terrains through the end of the mission in September 2016. Therefore this observation matches well with the erosion-driven activity that was escalating during the same period.

\subsection{Isolated patches close to irregular structures}

The isolated individual bright features that are located close to varying morphological structures fall into this category. These patches are often located under shadows of the neighbouring morphological structures and hence might be potentially able to survive for longer durations than their counterparts located on smooth terrains (Fig. 4). However, their lifetimes cannot be better constrained as they have not been imaged with favourable spatial resolutions and because of insufficient recurring observations. In the most favourable scenario, we are able to determine a lifetime of about 2 months for a patch in this category. Depending on the local solar zenith angle and neighbouring irregular structures, these features could be illuminated ephemerally. In the cases in which these features and their vicinity were favourably observed, these patches seem to appear on consolidated cometary material. 

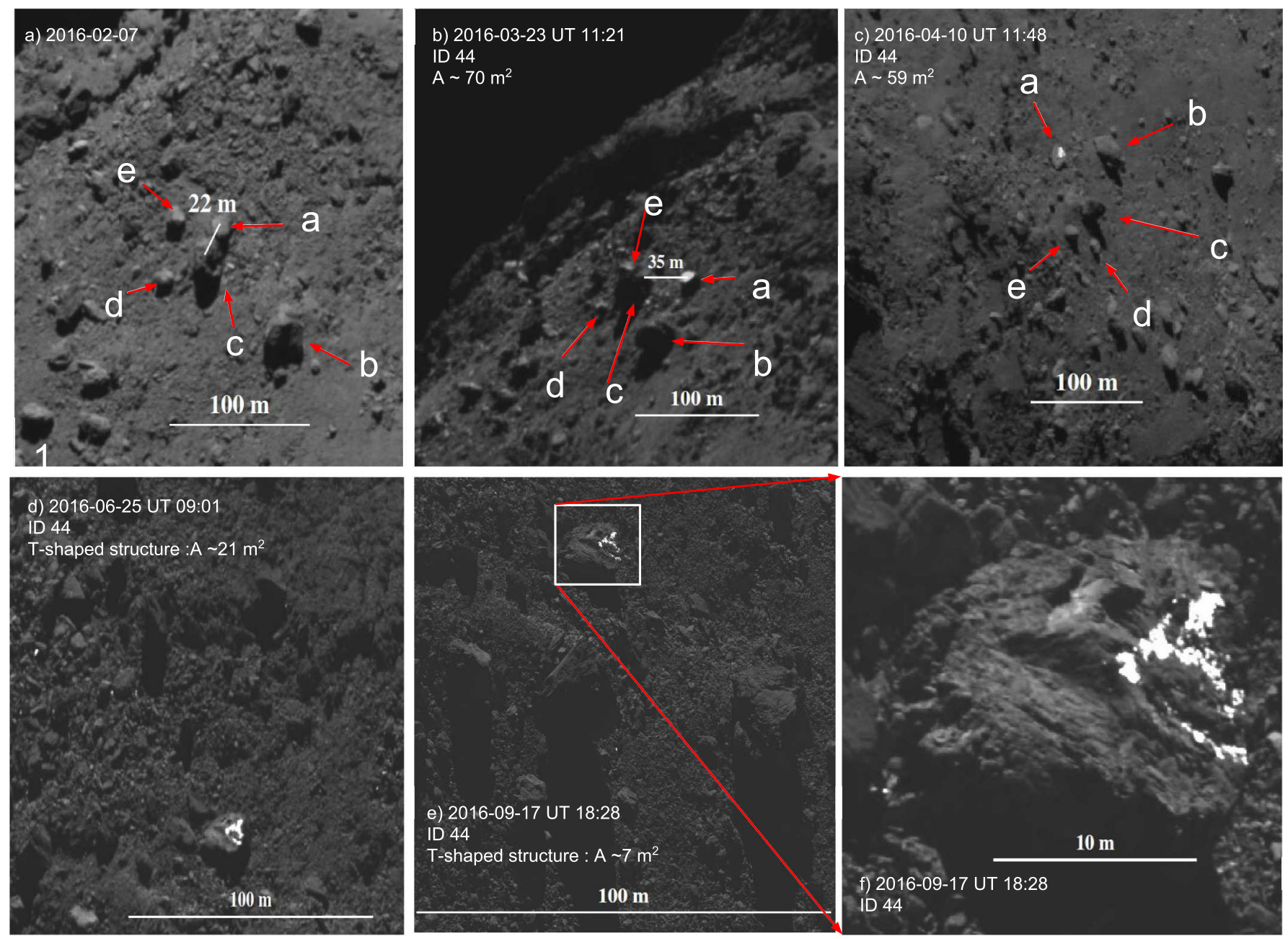

Fig. 5. Trigger and morphological evolution of a bright patch resting on a boulder in Bes region. The area measurements are approximative because of varying observational geometries.

Because of the consolidated nature of the material that hosts these features, they are hard to erode and hence the origin of these isolated patches is difficult to constrain.

We catalogue 18 patches of this type, which represents the largest number of bright patches belonging to any category. The appearance of such features does not seem to have any correlation with the heliocentric distance because they have been observed throughout the mission.

\subsection{Patches resting on boulders}

There have been a number of bright patches observed resting on the surface of boulders throughout the course of the mission (Fig. 4). Their presence on 67P was firstly highlighted by Pommerol et al. (2015) during early observations of the comet by Rosetta. In our framework, we catalogue a large number of such bright patches and suggest potential activity sources that might have triggered their appearance, based on OSIRIS observations.

There have been a few cases in which some cometary activity could be correlated to these boulders. Concerning a bright patch observed from January 2016 to July 2016 in the Khonsu region, a cometary outburst could be correlated with its location. This outburst took place in early August 2015 as the comet was approaching the perihelion of its orbit. As evident from Fig. 8, the available images of the location of the boulder taken before and after the perihelion show significant changes on the terrain, thus resulting in the appearance of the boulder with the bright patch on it as discussed by Deshapriya et al. (2016).

In addition to this, a displacement of a boulder can be observed in the Bes region. Comparing the geometry of a family of boulders in this region (Fig. 5), it can be established that some activity source has triggered a movement of a boulder between 7 February and 4 March 2016 (better constrained using NAVCAM images). Examining Fig. 5a and b, one could notice that the boulder annotated " $a$ " has a bright patch on it and both its distance and orientation relative to the centralised boulder " $b$ " have changed, which can also be validated using the co-ordinates of the boulders at aforementioned two dates. However, we are not able to determine the source of activity that triggered the displacement of this boulder. Nevertheless it is very likely that this same source has given rise to the appearance of the bright patch resting on the boulder. Further observations leading to the final stage of the mission reveal this patch in further detail because the spatial resolution significantly improved. The final observation of this patch dates to mid-September 2016 with a spatial resolution of about $6 \mathrm{~cm}$ per pixel and reveals several individual bright components forming an irregular shape. In March 2016, this patch appeared to be about $70 \mathrm{~m}^{2}$ and as the spatial resolution improved its structures could be resolved. On the final observation in mid-September, the patch appears to have an area of about $7 \mathrm{~m}^{2}$, indicating the nature of sublimation during the 6 months since its first detection. 

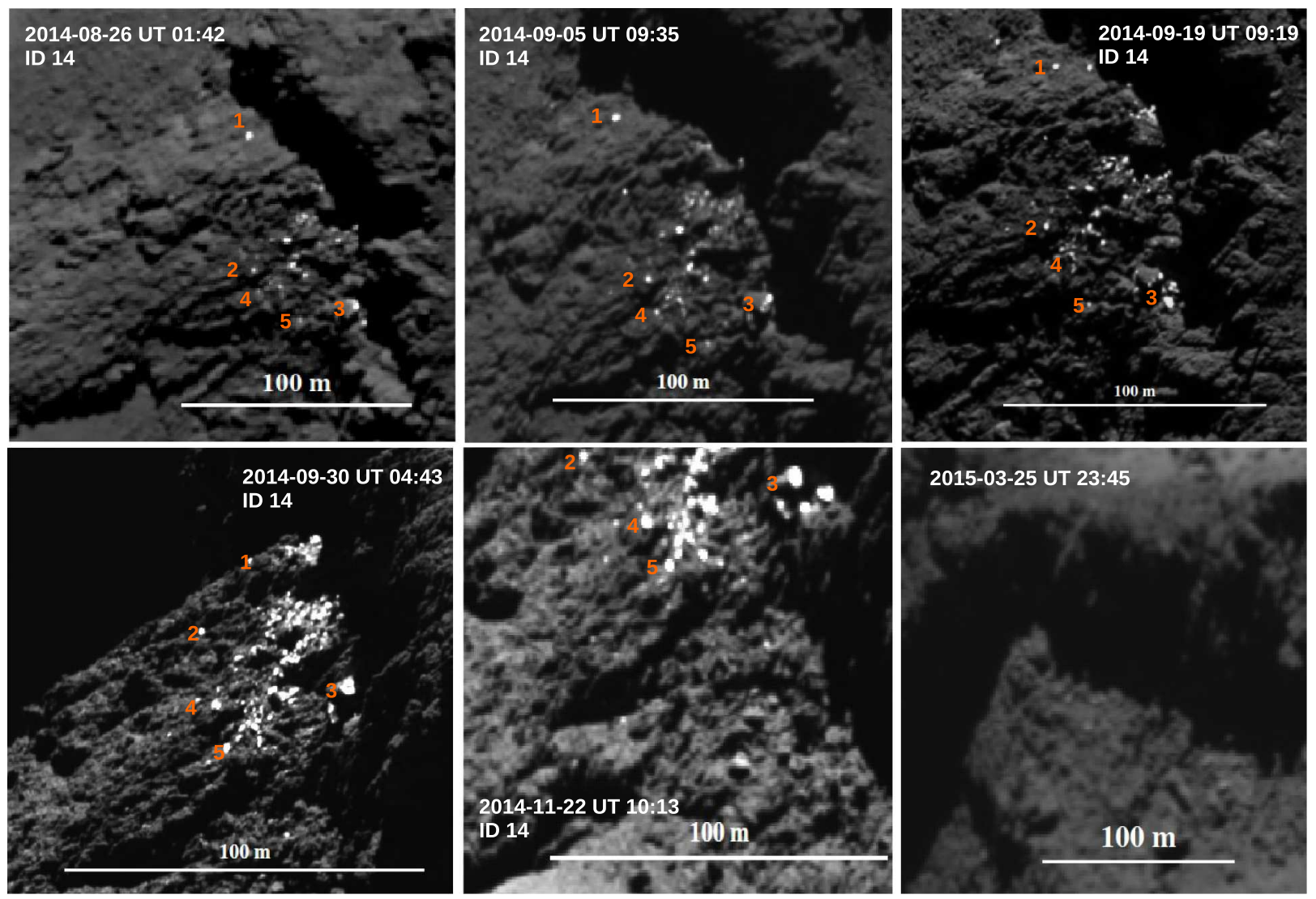

Fig. 6. Temporal variation of a cluster of bright patches in the Khepry region prior to the perihelion passage. Several individual patches of the cluster are numbered 1 through 5 to follow their stability over time. Despite varying spatial resolutions and observing geometries of the images, it is clear that the numbered patches have remained stable for about 3 months from end of August to end of November 2014. The latest of this sequence dating to end of March 2015 reveals no surviving patches and suggests their sublimation.

Although we are not able to trace any kind of activity for other patches resting on boulders probably because of the absence of observations during the transient activity time, supported by the above two cases, we suggest that they are correlated to sources of cometary activity.

\subsection{Clusters of patches}

There have been several observations of clusters of bright patches that are individually smaller in size compared to the aforementioned features. These clusters have mostly been observed in the pre-perihelion period and they are located near cliff structures. Therefore these clusters could be related to cliff collapse events, in which the resulting debris exposes water ice contained and preserved inside since their formation, as the major cliff collapse in Aswan area has been explained by Pajola et al. $(2015,2016)$.

Figure 6 shows a cluster of smaller bright features in the Khepry region. First observed at the end of August 2014, this cluster remained very stable for about 3 months. We numbered some of the individual patches to emphasise their stability in the sequence of images. This cluster might have remained stable for longer, but it is difficult to conclude owing to the inadequate temporal coverage of images. An observation dating back to 25 March 2015 indicates that the entire cluster has sublimated away, which indicates the influence of increasing solar irradiation on the clustered individual patches. This cluster was firstly studied by Pommerol et al. (2015) and Barucci et al. (2016) attributed its presence to $\mathrm{H}_{2} \mathrm{O}$ using VIRTIS-M infrared spectra.

\subsection{Frost}

Frost has been observed since the arrival of Rosetta (De Sanctis et al. 2015) at 67P (3.6 AU) by VIRTIS spectro-imager and has since been continuously observed by OSIRIS up to the end of the mission $(3.8 \mathrm{AU})$ with a pronounced abundance towards the perihelion passage (Fornasier et al. 2016). This frost could be observed at the local dawn on the comet, where the sun light returns after about $6 \mathrm{~h}$. Thus, the shadows cast by different morphological structures get shorter as the sun moves towards the local azimuth, revealing the frost formed over the cometary night due to condensation of previously sublimated water ice. Frost could be observed for some minutes before being rapidly sublimated away, depending on the heliocentric distance of the comet. This process continues as long as the solar irradiation is strong enough to sublimate the frost. Once the comet is beyond the current snowline of the solar system, the diurnal water cycle of the comet ceases, leading to no further frost sublimation until the comet returns inside the snowline for its next perihelion passage. This diurnal cycle of water was more pronounced as 67P got closer to the perihelion, as water production rates on the comet rose due to the increasing solar irradiation. Hence, close to the perihelion, both inbound and outbound, frost was ephemerally visible on a given location during the local cometary morning.

It is also possible that the four types of bright features introduced earlier, contributed to the diurnal cycle of water on the comet as they have been constantly sublimating, thus liberating the amount of water ice necessary to sustain the diurnal cycle of water on the comet. 
J. D. P. Deshapriya et al.: Exposed bright features on the comet 67P/Churyumov-Gerasimenko: distribution and evolution
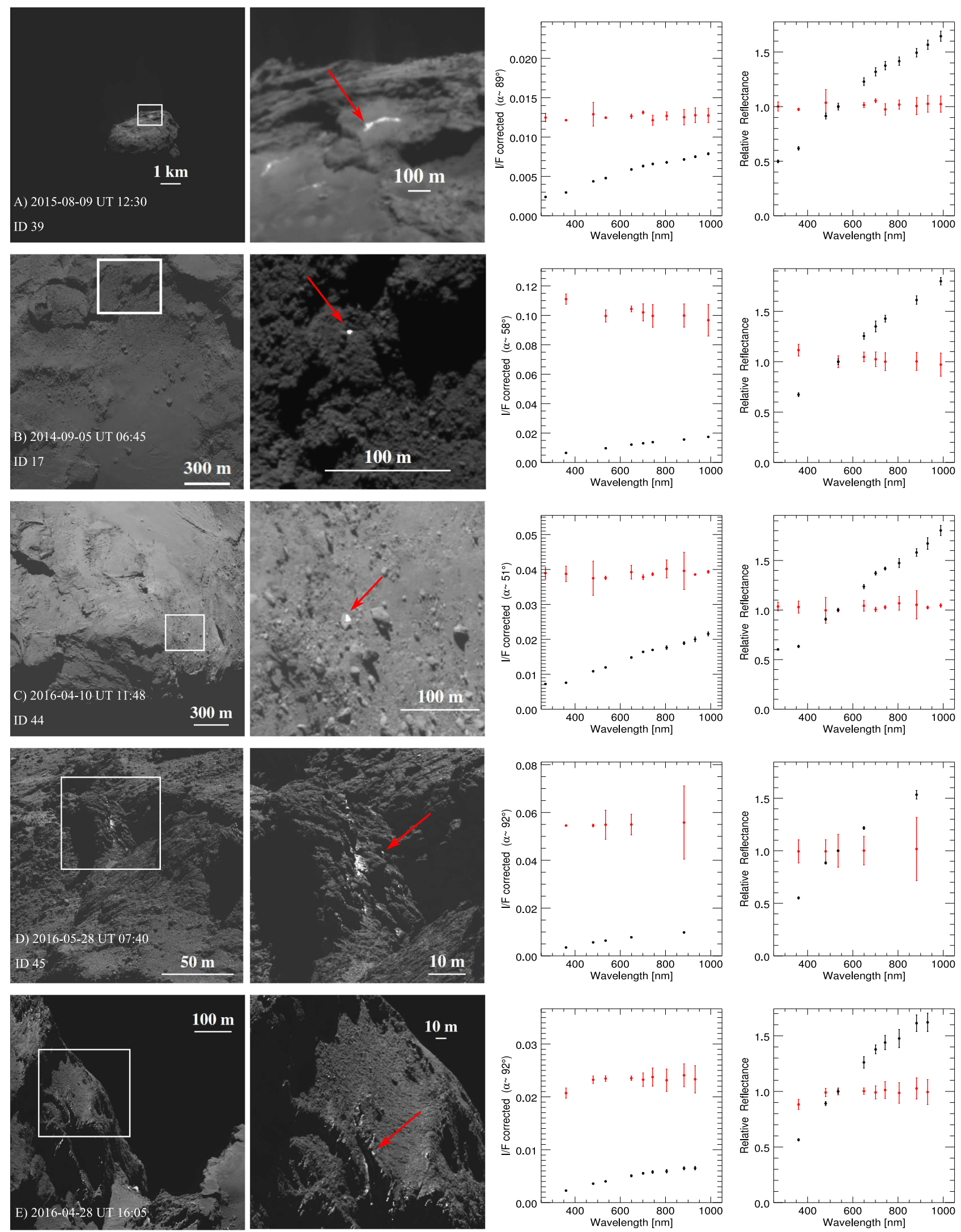

Fig. 7. Panels $A-D$ : four types of the studied bright features included in the catalogue and their spectrophotometric plots. Panel $E$ illustrates the frost and its spectrophotometric characteristics. Leftmost column shows a full-frame NAC ( $F 22$ filter centred at $649.2 \mathrm{~nm}$ ) image of the corresponding observations whereas the following 3 columns show a zoomed-in view of the bright feature; the corrected reflectance against the observed wavelength plot for the sampling area $(1 \times 2$ pixels for $\mathrm{A}$ through $\mathrm{D}$ and $2 \times 2$ pixels for $\mathrm{E})$ of the feature is indicated by the red arrow and a relative reflectance plot of the latter. The red dots correspond to the interested feature pointed by the arrow, whereas the black dots represent the cometary nucleus sampled from the vicinity of the interested feature. 

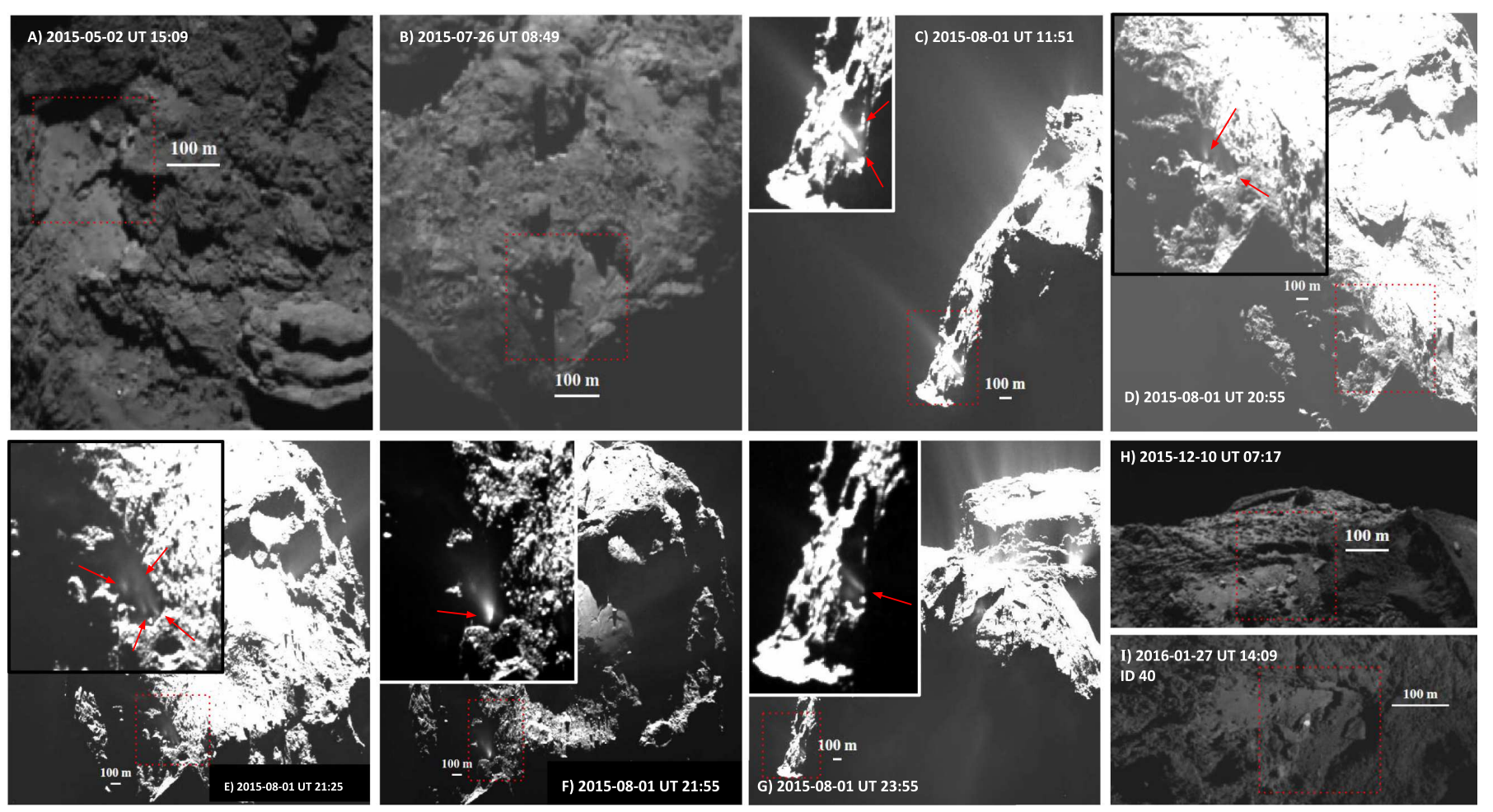

Fig. 8. Activity sources in the Khonsu region including a powerful outburst that modified the local terrain and later triggered the appearance of a bright patch resting on a boulder. Panel A shows the area of interest in the Khonsu region about 3 months prior to the perihelion passage. Panel $B$ corresponds to about three weeks before the perihelion passage and there are a couple of bright patches that have appeared on the smooth terrain probably due to the erosion that had been escalating at this time. The following panels from $C$ through $G$ illustrate the appearance of a family of transient outbursts and a long-lived outburst with a lifetime of the order of several hours, as the comet was accelerating towards the perihelion of its orbit. The next panel $\mathrm{H}$, where the area of interest is resolved about 4 months later, provides evidence that there have been significant changes on the surface morphology. The observable boulder field is therefore likely to be an aftermath of the aforementioned outbursts. Finally the panel $I$ reveals the bright patch that appeared on the boulder, some 2 months later.

\section{Spectrophotometry and albedo of five types of features}

We carried out spectrophotometric analyses for all the exposed bright features, which had multi-filter observations (51 out of 57 features). For each of the feature types, we selected a representative example from our catalogue along with frost. Reflectance $(I / F)$ plots and relative reflectance plots (normalised to the filter at $535 \mathrm{~nm}$ ) for each of the five types are presented in Fig. 7. The red dots correspond to the bright feature indicated with a red arrow, whereas the black dots are representative of the typical nucleus sampled from the vicinity. For each given panel, a NAC full-frame image is given followed by a zoom-in to the feature and the corresponding spectrophotometric plots.

For a given wavelength, the $I / F$ plots give an idea of the albedo of the feature compared to the typical 67P nucleus, which has a red spectral behaviour (increase of reflectance with the wavelength) due to the presence of the desiccated organics on the comet (Capaccioni et al. 2015). The different values of the $I / F$ of the exposed bright features in comparison to the $I / F$ of the typical cometary nucleus are connected to their varying ice contents. The relative $I / F$ curves of the bright features have a flat behaviour, as their relative reflectance stays more or less the same as the wavelength varies, thus suggesting the presence of ices. Although these ices could be composed of, for example $\mathrm{H}_{2} \mathrm{O}, \mathrm{CO}_{2}, \mathrm{CO}$, and $\mathrm{CH}_{4}$, we are not able to explicitly identify their composition with OSIRIS observations alone without complimentary spectroscopic data in the infrared range.
One cannot use the heliocentric distances corresponding to these observations to rule out the volatile ices that would have already sublimated because the comet could have irregular local insolation conditions dominated by seasonal and diurnal effects because of its very irregular shape. However there have been several studies (Barucci et al. 2016; Filacchione et al. 2016a; De Sanctis et al. 2015) based on VIRTIS spectroscopic data that confirm the presence of $\mathrm{H}_{2} \mathrm{O}$ ice on the comet compared to only one localised identification of $\mathrm{CO}_{2}$ ice (Filacchione et al. 2016b). Thus, $\mathrm{H}_{2} \mathrm{O}$ and $\mathrm{CO}_{2}$ are the only ices that have been spectroscopically identified on the comet 67P up to now. Given this context and OSIRIS spectrophotometric observations, we suggest that the observed ices are most likely to be $\mathrm{H}_{2} \mathrm{O}$ ice.

In order to compute geometric albedos for the exposed bright features, we relied on the phase curve of bright features presented in Hasselmann et al. (2017) using the OSIRIS WAC data of the Rosetta fly-by of 67P from 9 to 10 April 2016. During our calculation the effect of phase reddening of bright features was not considered under the assumption that its effect is negligible given the very low spectral slope values of such exposed bright features. We also note that the observations that corresponded to high phase angles (usually beyond $80^{\circ}$ ) were discarded due to the limited applicability of the aforementioned phase curve of bright features. The isolated patches on smooth terrains had albedos that ranged from $15 \%$ to $30 \%$, while the isolated patches close to irregular structures had albedos varying from $20 \%$ to $50 \%$. The albedos for the patches resting on boulders varied in the range of $15 \%$ to $75 \%$, whereas the clusters of patches had albedos that 
ranged from $10 \%$ to $50 \%$. It is evident from this analysis that the isolated patches on smooth terrains have the lowest upper limit of the albedos while the patches resting on boulders have the highest upper limit of albedos.

\section{Discussion}

We catalogue four types of exposed bright features on the comet. Type 1 are isolated patches located on smooth terrains of the comet and are highly correlated with the erosion that escalated towards the perihelion passage of 67P. Consequently almost all such features were observed within a period of 5 months before the perihelion passage. During this period we observe arc-shaped structures in Imhotep and Hapi regions and significantly large roundish patches in Anhur/Bes regions (Fornasier et al. 2016) all appearing on smooth terrains. It appears that these smooth terrains are covering the volatiles that are closest to the cometary surface. Assuming a dust-fall based formation for the smooth terrains during the previous perihelion passage, this type of volatiles could be formed through the cessation of the diurnal water cycle of the comet as the frost would not be sublimated any more as a result of gradually increasing heliocentric distances as the comet went pass beyond its perihelion. Nevertheless, we observed cometary frost and its sublimation even at 3.8 AU in the outbound orbit during the final stages of the mission. Hence, we could conclude that the cessation of the water cycle on the comet takes place somewhere between 3.8 AU and the aphelion of the comet $5.68 \mathrm{AU}$ in the outbound orbit. Consequently, a thin frost layer would be deposited on the nucleus, which would then be subject to dust fall, which would successively cover the frost layer. As the comet returns to the perihelion, passing again the snow line, when the incoming solar irradiation is adequate enough for sublimation to work, aforementioned frost layers could be observed as the dust would be lifted off and driven away by the sublimation-driven erosion. Further, the albedos of the features in this category suggest some mixing probably with cometary dust since they have the lowest upper limit of albedos among all the types of features. Type 2 are isolated patches close to irregular structures, which have been observed throughout the mission without any preferable heliocentric distance. Because of the local terrain and topography, these features seem to be often cast in shadows, consequently making them rarely observable. Type 3 are patches resting on boulders, which have been observed throughout the mission as well. For two patches of this Type 3 category, we find convincing evidence of cometary activity sources that have possibly triggered their appearance. These patches are also peculiar in the sense that they have longer lifetimes despite being regularly subject to insolation. Therefore, we could assume that some cometary activity source has modified the surface of the boulder, such that an internal reservoir of $\mathrm{H}_{2} \mathrm{O}$ ice could be exposed. In Fig. 5f we visualise the bright patch that has been imaged at the best spatial resolution, which is $6 \mathrm{~cm}$ per pixel. This is also the type of feature that corresponds to the highest upper limit of albedos among all the four types, which suggests that these patches are richer in ice content compared to the others. Type 4 are clusters of patches that show long-term stability at larger heliocentric distances in both inbound and outbound phases of the orbit of 67P. They are seldom observed close to the perihelion probably because they are too small to last long enough to be observed under escalated sublimation rates.

Figure 8 shows the surface evolution of a part of the Khonsu region bordering Imhotep and Apis regions. In each subframe the region of interest is indicated in a dotted square. As seen in
Fig. 8A, this region of interest is mainly characterised by smooth terrain, few surrounding boulders, and a terrace. The transition into panel 8B indicates the presence of two small bright patches, revealed probably due to the escalated erosion since the observation was about a couple of weeks before the perihelion passage. One of these is under the shadow of a neighbouring boulder and the spectrophotometry of these two patches hints at the presence of $\mathrm{H}_{2} \mathrm{O}$ ice. Panel $8 \mathrm{C}$ brings out a distinguishable collimated outburst (Vincent et al. 2016), originating from the location of the aforementioned bright patch under the shadow. The image is stretched to outline the outburst and zoomed view of the originating region reveals the presence of another minioutburst as indicated by the red arrow. Panel 8D shows the same region imaged $9 \mathrm{~h}$ after the main outburst event and more smallscale outbursts are identifiable. Panels $8 \mathrm{E}$ and $8 \mathrm{~F}$ show temporal variation of this family of outbursts and panel $8 \mathrm{G}$ depicts the long-lasting presence of the main outburst, some $12 \mathrm{~h}$ later the original detection. Imaged 4 months later, panel $8 \mathrm{H}$ shows what remains of the original terrain after the perihelion passage. The terrace structure has completely disappeared and we are able to identify several boulders scattered on the smooth terrain. About six weeks later panel 8I shows the presence of a bright patch (Deshapriya et al. 2016) resting on one of the boulders and in terms of the available data, we are not able to attribute any triggering activity source for this observation. The presence of this bright patch can be monitored for about 6 months as it was gradually being sublimated. Thus Fig. 8 shows the varying sources of activities and their successive aftermaths that took place in this terrain in the Khonsu region.

All the features presented in the catalogue in Appendix A are suggested to be attributed to $\mathrm{H}_{2} \mathrm{O}$ ice, relying on their spectrophotometry or photometry (in the event of absence of multi-filter observations). We also note the presence of frost on the comet throughout the mission, and there is a pronounced abundance towards the perihelion, where the $\mathrm{H}_{2} \mathrm{O}$ production rates increased.

\section{Conclusions}

The presented four different categories of exposed bright features on comet $67 \mathrm{P}$ have a spectral behaviour and temporal variations that are related to the presence of the volatile ices. However, based on the already available data corresponding to spectroscopic identification of ices on the nucleus of the comet $67 \mathrm{P}$, we suggest that the ice we report here are most likely to be $\mathrm{H}_{2} \mathrm{O}$ ice. Whatever the trigger of the formation of these different categories, their observation suggests that $\mathrm{H}_{2} \mathrm{O}$ ice is omnipresent inside the comet, hidden beneath dust layers and inside the consolidated cometary material. The fact that about $75 \%$ of all the features were observed prior to the perihelion passage suggests that increasing insolation has led to their exposure in different forms of cometary activity sources such as erosion and outbursts. Indeed seasonal colour changes indicate that the cometary dust mantle got thinner approaching the perihelion passage (Fornasier et al. 2016). We mapped all the studied exposed bright features onto a cylindrical projection of the comet and almost all of these features fall into the equatorial regions (between $-30^{\circ}$ and $+30^{\circ}$ of cometary latitude). Further, we also remark that a majority of them are concentrated in the southern latitudes.

Our analysis also suggests that the isolated bright $\mathrm{H}_{2} \mathrm{O}$ patches found on smooth terrains are not as freshly exposed as the other bright features catalogued in the sense that the former 
was formed after the previous perihelion passage of the comet as an aftermath of the cessation of the diurnal water cycle as the comet was getting further away from the sun. On the other hand, we suggest that the $\mathrm{H}_{2} \mathrm{O}$ ice on bright patches resting on boulders and on those found in clusters following a cliff collapse origin are freshly exposed because they are most likely to have remained inside the consolidated cometary material until the activity event that triggered their exposure. The fact that these patches also resulted in saturated pixels in many observations while the patches on smooth terrains did not supports the idea that the $\mathrm{H}_{2} \mathrm{O}$ ice found on bright patches resting on boulders and on clusters is less contaminated than $\mathrm{H}_{2} \mathrm{O}$ ice found on smooth terrains. This suggestion is additionally supported by the albedo calculations because the isolated patches on smooth terrains have the lowest upper limit of albedos $(30 \%)$, whereas the other three types have higher upper limits of albedo $(50 \%, 70 \%)$. Therefore we conclude that activity sources such as sublimation-driven erosion could only contribute to the appearance of bright patches on smooth terrains, whereas more powerful activity sources such as outbursts are capable of giving rise to other bright features containing a higher quantity of $\mathrm{H}_{2} \mathrm{O}$ ice as seen on boulders and cliff collapses.

Acknowledgements. OSIRIS was built by a consortium of the Max-PlanckInstitut für Sonnensystemforschung, Göttingen, Germany, CISAS-Università di Padova, Italy, the Laboratoire d'Astrophysique de Marseille, France, the Instituto de Astrofísica de Andalucia, CSIC, Granada, Spain, the Research and Scientific Support Department of the European Space Agency, Noordwijk The Netherlands, the Instituto Nacional de Técnica Aeroespacial, Madrid, Spain, the Universidad Politéchnica de Madrid, Spain, the Department of Physics and Astronomy of Uppsala University, Sweden, and the Institut für Datentechnik und Kommunikationsnetze der Technischen Universität Braunschweig, Germany. The support of the national funding agencies of Germany (DLR), France (CNES), Italy (ASI), Spain (MEC), Sweden (SNSB), and the ESA Technical Directorate is gratefully acknowledged. Rosetta is an ESA mission with contributions from its member states and NASA. All Rosetta NAVCAM images are released under the Creative Commons license ESA/Rosetta/ NAVCAM - CC BY-SA IGO 3.0.

\section{References}

Barucci, M. A., Filacchione, G., Fornasier, S., et al. 2016, A\&A, 595, A102

Bertin, E. 2012, in ASP Conf. Ser., 461, eds. P. Ballester, D. Egret, \& N. P. F Lorente, 263

Capaccioni, F., Coradini, A., Filacchione, G., et al. 2015, Science, 347, aaa0628

De Sanctis, M. C., Capaccioni, F., Ciarniello, M., et al. 2015, Nature, 525, 500

Deshapriya, J. D. P., Barucci, M. A., Fornasier, S., et al. 2016, MNRAS, 462, S274

El-Maarry, M. R., Groussin, O., Thomas, N., et al. 2017, Science, 355, 1392

Fairbairn, M. B. 2005, J. R. Astron. Soc. Can., 99, 92

Fanale, F. P., \& Salvail, J. R. 1984, Icarus, 60, 476

Filacchione, G., De Sanctis, M. C., Capaccioni, F., et al. 2016a, Nature, 529, 368

Filacchione, G., Raponi, A., Capaccioni, F., et al. 2016b, Science, 354, aag3161

Fornasier, S., Hasselmann, P. H., Barucci, M. A., et al. 2015, A\&A 583, A30

Fornasier, S., Mottola, S., Keller, H. U., et al. 2016, Science, 354, 1566

Fornasier, S., Feller, C., Lee, J.-C., et al. 2017, MNRAS, 469, S93

Groussin, O., Sierks, H., Barbieri, C., et al. 2015a, A\&A, 583, A36

Groussin, O., Jorda, L., Auger, A.-T., et al. 2015b, A\&A, 583, A32

Hasselmann, P. H., Barucci, M. A., Fornasier, S., et al. 2017, MNRAS, 469, S550

Jorda, L. Gaskell, R., Capanna, C., et al. 2016, Icarus, 277, 257

Keller, H. U., Barbieri, C., Lamy. P., et al. 2007, Space Sci. Rev., 128, 26

Küppers, M., Mottola, S., Lowry, S. C., et al. 2007, A\&A, 462, 13

Oklay, N., Vincent, J.-B., Fornasier, S., et al. 2016, A\&A, 586, A80
Oklay, N., Mottola, S., Vincent, J.-B., et al. 2017, MNRAS, 469, S582 Pajola, M., Vincent, J.-B., Güttler, C., et al. 2015, A\&A, 583, A37 Pajola, M., Oklay, N., La Forgia, F., et al. 2016, A\&A, 592, A69 Pajola, M., Höfner, S., Vincent, J. B., et al. 2017, Nat. Astron., 1, 0092 Pommerol, A., Thomas, N., El-Maarry, M. R., et al. 2015, A\&A, 583, 25 Preusker, F., Scholten, F., Matz, K.-D., et al. 2015, A\&A, 583, A33 Sears, D. W. G., Kochan, H. W., \& Huebner, W. F. 1999, Meteor. Planet. Sci., 34 497

Sierks, H., Barbieri, C., Lamy, P. L., et al. 2015, Science, 347, aaa1044 Thomas, N., Sierks, H., Barbieri, C., et al. 2015, Science, 347, aaa0440 Tubiana, C., Güttler, C., Kovacs, G., et al. 2015, A\&A, 583, A46

Vincent, J.-B., A'Hearn, M. F., Lin, Z.-Y., et al. 2016, MNRAS, 462, S184

1 LESIA, Observatoire de Paris, PSL Research University, CNRS, Univ. Paris Diderot, Sorbonne Paris Cité, UPMC Univ. Paris 06, Sorbonne Universités, 5 place Jules Janssen, 92195 Meudon, France e-mail: prasanna. deshapriya@obspm. fr

2 Max-Planck-Institut für Sonnensystemforschung, Justus-vonLiebig-Weg 3, 37077 Göttingen, Germany

3 INAF-OAPD, Astronomical Observatory of Padova, Vicolo dell'Osservatorio 5, 35122 Padova, Italy

4 NASA Ames Research Center, Mountain View, CA 94035, USA

5 Institut für Planetenforschung, DLR, Rutherfordstrasse 2, 12489 Berlin, Germany

6 Dipartimento di Fisica e Astronomia "Galileo Galilei", Università di Padova, Vic. Osservatorio 3, 35122 Padova, Italy

7 Laboratoire d'Astrophysique de Marseille UMR 7326, CNRS, AixMarseille Université, 13388 Marseille Cedex 13, France

8 Centro de Astrobiología, CSIC-INTA, 28850 Torrejón de Ardoz, Madrid, Spain

9 International Space Science Institute, Hallerstrasse 6, 3012 Bern, Switzerland

10 Research and Scientific Support Department, European Space Agency, 2201 Noordwijk, The Netherlands

11 Department of Physics and Astronomy, Uppsala University, 75120 Uppsala, Sweden

12 Polish Academy of Sciences, Space Research Center, Bartycka 18A, 00716 Warszawa, Poland

13 LATMOS, CNRS/UVSQ/IPSL, 11 Boulevard d'Alembert, 78280 Guyancourt, France

14 Department of Astronomy, University of Maryland, College Park, MD 20742-2421, USA

15 CNR-IFN UOS Padova LUXOR, Via Trasea 7, 35131 Padova, Italy

16 NASA Jet Propulsion Laboratory, 4800 Oak Grove Drive, Pasadena, CA 91109, USA

17 Dipartimento di Ingegneria Meccanica, Università di Padova, Via Venezia 1, 35131 Padova, Italy

18 UNITN, Universitá di Trento, Via Mesiano 77, 38100 Trento, Italy

19 INAF - Osservatorio Astronomico di Trieste, Via Tiepolo 11, 34143 Trieste, Italy

20 Instituto de Astrofísica de Andalucía - CSIC, 18080 Granada, Spain

21 Institute for Space Science, National Central University, 32054 Chung-Li, Taiwan

22 Institut für Geophysik und Extraterrestrisch Physik, TU Braunschweig, 38106 Braunschweig, Germany

23 ESA/ESAC, PO Box 78, 28691 Villanueva de la Cañada, Spain

24 Centro di Ateneo di Studi e Attività Spaziali "Giuseppe Colombo" (CISAS), Università di Padova, Via Venezia 15, 35131 Padova, Italy

25 Dipartimento di Ingegneria dell'Informazione, Università di Padova, Via Gradenigo 6/B, 35131 Padova, Italy

26 Physikalisches Institut, Sidlerstrasse 5, Universität Bern, 3012 Bern, Switzerland 


\section{Appendix A: Catalogue of exposed bright features}

Table A.1. Catalogue of exposed bright features on comet 67P.

\begin{tabular}{|c|c|c|c|c|c|c|c|c|c|c|c|}
\hline ID & $\begin{array}{l}\text { Detection } \\
\text { (UT) }\end{array}$ & Type & $\begin{array}{c}A \\
\left(\mathrm{~m}^{2}\right)\end{array}$ & $\begin{array}{r}\text { Life } \\
\text { (days) }\end{array}$ & Region & $\begin{array}{c}\text { Phase } \\
\left({ }^{\circ}\right)\end{array}$ & $\begin{array}{c}\text { Res. } \\
\text { (m/pixel) }\end{array}$ & $\begin{array}{r}\text { Lat. } \\
\left({ }^{\circ}\right)\end{array}$ & $\begin{array}{r}\text { Long. } \\
\left({ }^{\circ}\right)\end{array}$ & Obs. & Remarks \\
\hline 1 & 2014-08-05 23:19 & 3 & 50.6 & 123 & Wosret & 48.5 & 2.25 & -7.8 & 356.3 & NAC & \\
\hline 2 & 2014-08-06 00:19 & 3 & 48.8 & 129 & Ma'at & 49.8 & 2.19 & 14.2 & 356.7 & NAC & P Fig. 4d \\
\hline 3 & 2014-08-06 02:43 & 4 & - & 44 & Anuket & 51.0 & 2.17 & -1.4 & 304.7 & NAC & P Fig. 1d \\
\hline 4 & 2014-08-14 08:43 & 2 & 12.4 & 36 & Aker & 35.7 & 1.76 & -3.7 & 61.4 & NAC & \\
\hline 5 & 2014-08-14 08:43 & 3 & 31.0 & 154 & Atum & 35.7 & 1.76 & -26.7 & 232.3 & NAC & P Fig. 4b \\
\hline 6 & 2014-08-16 14:59 & 4 & - & 98 & Imhotep & 38.9 & 1.84 & 13.4 & 116.5 & NAC & Fi(a) BAP 1 \\
\hline 7 & 2014-08-16 15:59 & 4 & - & 98 & Imhotep & 39.4 & 1.86 & -2.7 & 180.6 & NAC & Fi(a) BAP 2 \\
\hline 8 & 2014-08-22 16:42 & 2 & 49.9 & 12 & Ash & 34.0 & 1.09 & 21.1 & 200.9 & NAC & \\
\hline 9 & 2014-08-22 16:42 & 2 & 22.5 & 1 & Ash & 34.03 & 1.09 & 36.4 & 136.8 & NAC & \\
\hline 10 & 2014-08-23 07:44 & 2 & 13.0 & 91 & Imhotep & 40.1 & 1.09 & -22.0 & 182.8 & NAC & B Spot 5, O Fig. 9, P Fig. 7a \\
\hline 11 & 2014-08-23 12:42 & 2 & 74.0 & 10 & Geb & 47.2 & 1.12 & -48.3 & 295.8 & NAC & \\
\hline 12 & 2014-08-25 19:12 & 4 & - & 133 & Atum & 37.5 & 0.93 & -20.7 & 227.4 & NAC & B Spot 4 \\
\hline 13 & 2014-08-25 23:42 & 3 & 21.5 & 36 & Imhotep & 37.7 & 0.93 & -2.4 & 174.8 & NAC & B Spot 8 \\
\hline 14 & 2014-08-26 01:42 & 4 & - & 88 & Khepry & 38.1 & 0.94 & 4.2 & 71.7 & NAC & B Spot 6, P Fig. 7b \\
\hline 15 & 2014-09-02 02:42 & 4 & - & 29 & Ash & 38.5 & 0.94 & 34.7 & 137.2 & NAC & P Fig. 1c \\
\hline 16 & 2014-09-05 05:21 & 4 & - & 146 & Imhotep & 57.2 & 0.77 & -8.1 & 188.3 & NAC & B Spot 7 \\
\hline 17 & 2014-09-05 06:45 & 2 & 8.6 & 0 & Ash & 57.8 & 0.76 & 22.9 & 122.9 & NAC & \\
\hline 18 & 2014-09-15 05:42 & 3 & 4.2 & 0 & Bastet & 67.35 & 0.51 & -1.1 & 5.2 & NAC & \\
\hline 19 & 2014-09-15 20:32 & 3 & 13.8 & 69 & Maftet & 73.4 & 0.50 & -15.1 & 312.5 & NAC & P Fig. 4c \\
\hline 20 & 2015-01-04 02:22 & 3 & 29.0 & 58 & Khonsu & 95.3 & 0.53 & -23.8 & 198.3 & NAVCAM & B Spot 3, De Patch 1 \\
\hline 21 & 2015-03-10 21:55 & 1 & 35.8 & 33 & Bes & 55.1 & 1.6 & -30.2 & 260.8 & NAC & \\
\hline 22 & 2015-03-21 & 1 & - & 42 & Anhur & - & - & -54.5 & 66.0 & VIRTIS & $\mathrm{Fi}(\mathrm{b})$ \\
\hline 23 & 2015-03-25 10:42 & 4 & - & 16 & Khepry & 73.4 & 1.67 & -0.2 & 75.8 & NAC & \\
\hline 24 & $2015-04-27$ 15:58 & 1 & 277.3 & 10 & Bes & 73.13 & 2.43 & -54.6 & 75.6 & NAC & Fo16 \\
\hline 25 & $2015-05-02$ 05:53 & 2 & 42.2 & 0 & Bes & 61.57 & 2.29 & -84.6 & 218.2 & NAC & \\
\hline 26 & 2015-05-07 22:06 & 2 & 132.1 & 0 & Bes & 61.01 & 2.57 & -86.7 & 243.6 & NAC & \\
\hline 27 & 2015-06-04 23:16 & 2 & 104.1 & 37 & Anhur & 87.42 & 3.86 & -41.7 & 63.7 & NAC & B Spot 2 \\
\hline 28 & 2015-06-05 07:03 & 2 & 220.0 & 22 & Imhotep & 87.2 & 3.83 & -5.8 & 189.4 & NAC & B Spot 1 \\
\hline 29 & 2015-06-13 15:20 & 1 & 13.6 & 21 & Imhotep & 87.8 & 3.69 & 10.6 & 170.8 & NAC & \\
\hline 30 & 2015-06-24 07:59 & 1 & 76.9 & 25 & Hapi & 89.7 & 3.24 & -8.0 & 260.4 & NAC & $\mathrm{Da}$ \\
\hline 31 & 2015-06-27 08:33 & 1 & 204.2 & 29 & Serqet & 89.7 & 3.69 & 7.7 & 312.5 & NAC & \\
\hline 32 & 2015-06-27 15:08 & 1 & 51.3 & 22 & Imhotep & 89.5 & 3.58 & -10.7 & 135.5 & NAC & \\
\hline 33 & 2015-07-04 08:58 & 1 & 98.6 & 0 & Khepry & 89.9 & 3.31 & -12.7 & 91.3 & NAC & \\
\hline 34 & 2015-07-04 13:41 & 1 & 74.9 & 15 & Hatmehit & 89.8 & 3.27 & 9.1 & 346.9 & NAC & \\
\hline 35 & 2015-07-11 13:41 & 1 & 139.2 & 0 & Imhotep & 89.6 & 2.95 & 3.9 & 131.1 & NAC & \\
\hline 36 & 2015-07-11 13:41 & 1 & 78.3 & 0 & Imhotep & 89.6 & 2.95 & 10.4 & 156.7 & NAC & \\
\hline 37 & 2015-07-11 13:41 & 2 & 52.2 & 0 & Imhotep & 89.6 & 2.95 & 12.6 & 178.8 & NAC & \\
\hline 38 & 2015-07-15 15:58 & 1 & 36.7 & 11 & Imhotep & 89.5 & 3.03 & -0.8 & 185.3 & NAC & \\
\hline 39 & 2015-07-26 12:11 & 1 & 903 & 14 & Imhotep & 89.4 & 3.88 & -29.1 & 110.3 & NAC & \\
\hline 40 & 2016-01-23 23:45 & 3 & 58.0 & 188 & Khonsu & 61.2 & 1.35 & -13.3 & 196.4 & NAC & De Patch 2 \\
\hline 41 & 2016-01-28 03:48 & 2 & 27.2 & 0 & Imhotep & 62.0 & 1.23 & 20.1 & 139.9 & NAC & \\
\hline 42 & 2016-02-10 11:53 & 2 & 28.7 & 0 & Khonsu & 65.2 & 0.89 & -18.7 & 196.4 & NAC & De Patch 3 \\
\hline 43 & 2016-03-23 07:59 & 4 & - & 115 & Khonsu & 91.6 & 0.19 & -24.2 & 200.4 & NAC & De Patch 4 \\
\hline 44 & 2016-03-23 11:21 & 3 & 70.0 & 178 & Bes & 106.0 & 1.08 & -31.8 & 86.3 & NAC & \\
\hline
\end{tabular}

Notes. Column description: identification number of the exposed bright feature, first detection time, type of feature (1-4), area of feature, lifetime of feature, phase angle at the time of the first detection, spatial resolution, latitude, longitude, observing instrument, and remarks that refer to other mentions of the same feature by different publications. The legend of publications in the Remarks column is as follows: B: Barucci et al. (2016), Da: Davidsson et al. (in prep.), De: Deshapriya et al. (2016), Fi(a): Filacchione et al. (2016a), Fi(b): Filacchione et al. (2016b), Fo16: Fornasier et al. (2016), Fo17: Fornasier et al. (2017), O: Oklay et al. (2016), P: Pommerol et al. (2015). 
A\&A 613, A36 (2018)

Table A.1. continued.

\begin{tabular}{|c|c|c|c|c|c|c|c|c|c|c|c|}
\hline ID & $\begin{array}{l}\text { Detection } \\
(\mathrm{UT})\end{array}$ & Type & $\begin{array}{c}A \\
\left(\mathrm{~m}^{2}\right) \\
\end{array}$ & $\begin{array}{r}\text { Life } \\
\text { (days) }\end{array}$ & Region & $\begin{array}{c}\text { Phase } \\
\left({ }^{\circ}\right)\end{array}$ & $\begin{array}{c}\text { Res. } \\
\text { (m/pixel) }\end{array}$ & $\begin{array}{r}\text { Lat. } \\
\left({ }^{\circ}\right) \\
\end{array}$ & $\begin{array}{r}\text { Long. } \\
\left({ }^{\circ}\right) \\
\end{array}$ & Obs. & Remarks \\
\hline 45 & 2016-04-29 04:42 & 4 & - & 50 & Aten & 92.1 & 0.31 & 19.5 & 104.2 & NAC & \\
\hline 46 & 2016-04-30 10:41 & 4 & - & 0 & Serqet & 90.5 & 0.30 & 7.7 & 311.8 & NAC & \\
\hline 47 & 2016-05-15 19:09 & 3 & 0.8 & 83 & Wosret & 90.3 & 0.14 & -7.7 & 357.5 & NAC & \\
\hline 48 & 2016-05-19 15:46 & 2 & 0.5 & 66 & Imhotep & 101.8 & 0.11 & -28.7 & 166.4 & NAC & \\
\hline 49 & 2016-06-14 03:37 & 3 & 7.2 & 0 & Khonsu & 57.3 & 0.49 & -15.2 & 199.5 & NAC & \\
\hline 50 & 2016-07-02 07:57 & 2 & 10.6 & 7 & Khonsu & 86.1 & 0.71 & -20.2 & 197.7 & NAC & \\
\hline 51 & 2016-07-09 02:42 & 3 & 1.26 & 0 & Babi & 89.6 & 0.30 & 6.3 & 70.1 & NAC & \\
\hline 52 & $2014-10-03$ 23:22 & 1 & 0.3 & 97 & Ash & 92.9 & 0.32 & 34.1 & 196.3 & NAC & P Fig. 3b \\
\hline 53 & 2014-10-04 19:16 & 2 & 1.0 & 0 & Bastet & 92.8 & 0.31 & 2.3 & 5.9 & NAC & P Fig. 3d \\
\hline 54 & $2014-10-05$ 23:54 & 3 & 3.1 & 0 & Maftet & 92.3 & 0.31 & -11.2 & 313.0 & NAC & P Fig. $4 a$ \\
\hline 55 & 2014-10-05 23:54 & 2 & 1.8 & 50 & Maftet & 92.3 & 0.31 & -10.5 & 324.7 & NAC & P Fig. 3a \\
\hline 56 & $2016-01-2805: 33$ & 2 & 13.4 & 0 & Anhur & 61.9 & 1.22 & -44.7 & 33.1 & NAC & Fo17 \\
\hline 57 & 2016-09-02 00:30 & 4 & - & 23 & Atum & 107.5 & 1.23 & -22.4 & 225.2 & WAC & \\
\hline
\end{tabular}

